\title{
Yersiniabactin from Yersinia pestis: biochemical characterization of the siderophore and its role in iron transport and regulation
}

\author{
Robert D. Perry, Paul B. Balbo, Heather A. Jones, \\ Jacqueline D. Fetherston and Edward DeMoll \\ Author for correspondence: Robert D. Perry. Tel: +1606323 6341. Fax: + 16062578994 \\ e-mail : rperry@pop.uky.edu
}

Department of Microbiology and Immunology, MS415 Medical Center, University of Kentucky, Lexington, KY 40536-0084, USA

\begin{abstract}
A siderophore-dependent iron transport system of the pathogenic yersiniae plays a role in the pathogenesis of these organisms. The structure of the yersiniabactin (Ybt) siderophore produced by Yersinia enterocolitica has been elucidated. This paper reports the purification of Ybt from Yersinia pestis and demonstrates that it has the same structure as Ybt from Y. enterocolitica. Purified Ybt had a formation constant for $\mathrm{Fe}^{3+}$ of $\sim 4 \times 10^{-36}$. Addition of purified Ybt from $Y$. pestis enhanced iron uptake by a siderophore-negative (irp2) strain of $Y$. pestis. Maximal expression of the Ybt outer-membrane receptor, Psn, in this strain was dependent upon exogenously supplied Ybt. Regulation of Psn expression by Ybt occurred at the transcriptional level. $Y$. pestis DNA was used to construct irp2 and psn mutations in Yersinia pseudotuberculosis. The irp2 mutant strain no longer synthesized $Y b t$ and the psn mutant strain could not use exogenously supplied Ybt. As in Y. pestis, Ybt was required for maximal expression of Psn. Regulation by Ybt occurred at the transcriptional level. In contrast to $Y$. pestis, in which a psn mutation does not repress synthesis of Ybt siderophore or expression of the iron-regulated HMWP1 and HMWP2 proteins, the same mutation in Y. pseudotuberculosis partially repressed these products.
\end{abstract}

Keywords: plague, iron affinity, iron transport, siderophore

\section{INTRODUCTION}

Iron is an essential trace element for nearly all organisms. The exceedingly low solubility of ferric ions at neutral $\mathrm{pH}$ under aerobic conditions and the chelation of iron and haem by mammalian proteins requires microbial pathogens to elaborate high-affinity transport systems to acquire sufficient iron for growth. One solution to acquiring essential iron is the use of siderophore-dependent iron-transport systems. Siderophores are low-molecular-mass compounds with high affinities for ferric iron that are secreted into the environment, where they bind precipitated iron or remove it from host binding proteins. Uptake of the iron-siderophore complex provides the bacterial cell

Abbreviations: CCCP, carbonyl cyanide $m$-chlorophenylhydrazone; DIP, 2,2'-dipyridyl; HMWP, high molecular weight (mass) protein; MALDI, matrix-assisted laser desorption; OM, outer membrane; TBA, tryptose blood agar base; Ybt, yersiniabactin. with the iron needed for growth (Braun et al., 1998; Byers \& Arceneaux, 1998; Guerinot, 1994; Mietzner \& Morse, 1994).

The production of a siderophore-like activity by Yersinia pestis was reported over 20 years ago (Wake $e t$ al., 1975). Since then there have been extensive studies on this siderophore-dependent iron-transport system, known as the yersiniabactin (Ybt) system. The $\mathrm{Ybt}$ system is encoded within a high-pathogenicity island and is present in the pathogenic strains of Y. pestis, Yersinia pseudotuberculosis and Yersinia enterocolitica as well as several types of enteropathogenic Escherichia coli (Buchrieser et al., 1998; Carniel et al., 1996; de Almeida et al., 1993; Pelludat et al., 1998; Rakin et al., 1995; Schubert et al., 1998). For Y. pestis and Y. enterocolitica, nearly all the genes of the Ybt system have been sequenced (Bearden et al., 1997; Fetherston et al., 1995, 1996; Gehring et al., 1998a; Guilvout et al., 1993; Pelludat et al., 1998; Rakin et al., 1994); the 


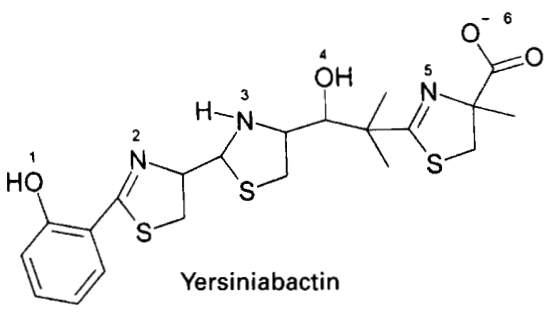<smiles>O=C(C1CSC(c2cccc(O)c2O)=N1)N(O)CCc1c[nH]cn1</smiles>

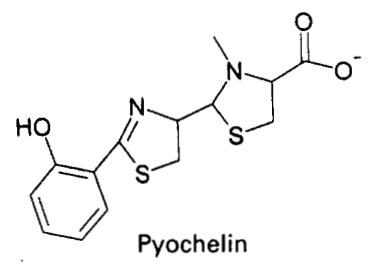

Fig. 1. Structure of yersiniabactin and the related siderophores anguibactin and pyochelin. Ferric-yersiniabactin shows the proposed iron coordination sites (indicated by small numbers).

$>97 \%$ sequence identity suggests that the system is functionally identical in these two pathogens. A number of genes thought to be involved in the biosynthesis of Ybt have been identified based on sequence similarities to other siderophore biosynthetic genes (Bearden et al., 1997; Gehring et al., 1998a; Guilvout et al., 1993; Pelludat et al., 1998). However, the function of only a small number of the putative Ybt biosynthetic genes has been conclusively demonstrated. Mutational analyses have shown that irp2 (encoding HMWP2, a nonribosomal peptide synthetase), $y b t E$ (encoding a salicylAMP ligase) and $y b t S$ (probably encoding an enzyme required for salicylate synthesis) are essential for $\mathrm{Ybt}$ biogenesis (Bearden et al., 1997; Gehring et al., 1998a). In addition, biochemical functions, predicted from sequence analysis, have been demonstrated for HMWP2, HMWP1 (encoded by irp1) and YbtE (Gehring et al., 1998a, b). Yersiniabactin produced by $Y$. enterocolitica, alternatively termed yersiniophore when the two activities were thought to be chemically distinct (Chambers \& Sokol, 1994), has been isolated and structurally characterized (Chambers et al., 1996; Drechsel et al., 1995; Haag et al., 1993). The Ybt molecule is composed of a phenolic group, probably derived from salicylate, as well as one thiazolidine and two thiazoline rings that are derived from the condensation of three cysteine residues (Gehring et al., 1998a). As previously noted (Chambers et al., 1996; Drechsel et al., 1995), this structure contains significant similarities to pyochelin and anguibactin, siderophores produced by Pseudomonas aeruginosa and Vibrio anguillarum respectively (Cox et al., 1981; Jalal et al., 1989) (Fig. 1).
Here we describe the purification and structural characterization of Ybt produced by $Y$. pestis, the causative agent of plague. Ybt from Y. pestis is identical to that from Y. enterocolitica and has a high affinity for ferric iron. Purified Ybt enhances iron uptake and expression of the $p s n$ gene that encodes the outer-membrane (OM) receptor for $\mathrm{Ybt}$. In addition, we have used mutations in $Y$. pestis irp2 and psn genes to generate similar mutations in $Y$. pseudotuberculosis. These studies indicate that the Ybt systems in $Y$. pestis and $Y$. pseudotuberculosis are interchangeable and that $\mathrm{Ybt}$ also participates in regulating gene expression in $Y$. pseudotuberculosis.

\section{METHODS}

Bacterial strains, plasmids and media. All relevant characteristics of strains and plasmids used in this study are presented in Table 1. Y. pseudotuberculosis strains with mutations in irp2 and $p s n$ were constructed using suicide vehicles pPSN15 and pCIRP498.8 for allelic exchange as previously described for Y. pestis (Fetherston et al., 1995). Mutations were confirmed by Southern blot analysis and by loss of pesticin sensitivity for the $\Delta p s n 2045.1$ mutation (Fetherston \& Perry, 1994) or by loss of Ybt siderophore production (see below). Y. pseudotuberculosis strains cured of the low-calcium-response virulence plasmid were selected by growth at $37^{\circ} \mathrm{C}$ on tryptose blood agar base (Difco) plates supplemented with $20 \mathrm{mM}$ sodium oxalate and $20 \mathrm{mM} \mathrm{MgCl}_{2}$ (Higuchi \& Smith, 1961). Plasmids were purified from overnight heart infusion broth (Difco) cultures by alkaline lysis (Birnboim \& Doly, 1979) and further purified when necessary by polyethylene glycol precipitation (Humphreys et al., 1975). Y. pestis and Y. pseudotuberculosis cells were transformed by electroporation as previously described (Fetherston et al., 1995). Where relevant, the haemin storage $\left(\mathrm{Hms}^{+}\right)$phenotype of $Y$. pestis strains was determined on Congo red plates (Surgalla \& Beesley, 1969). For studies under iron-starvation conditions, cells were cultivated at $37^{\circ} \mathrm{C}$ in the defined medium $\mathrm{PMH}$ deferrated by extraction with Chelex 100 (Staggs \& Perry, 1991). Precipitation of residual iron in deferrated PMH was achieved by supplementation with $0.5 \mathrm{mM} \mathrm{NaCO}_{3}, 0.01 \mathrm{mM}$ $\mathrm{MnCl}_{2}$ and 4.0 $\mathrm{mM} \mathrm{CaCl}_{2}$ (PMH-S) (Fetherston et al., 1995). PMH-S was solidified with $1 \%$ agarose. For Y. pseudotuberculosis studies, PMH-S plates were supplemented with the iron chelator 2,2'-dipyridyl (DIP). For growth under various iron conditions, acid-cleaned glassware was soaked overnight in chromic/sulfuric acid (46.3 $\mathrm{g} \mathrm{K}_{2} \mathrm{Cr}_{2} \mathrm{O}_{7}$ per litre $12 \mathrm{M}$ sulfuric acid) or Dichrol (Baxter Diagnostics) to remove contaminating iron and copiously rinsed in deionized water.

Protein, $\beta$-galactosidase and pesticin sensitivity analyses. Whole cells of Y. pestis and Y. pseudotuberculosis strains, subjected to iron-deficient or iron-sufficient growth conditions in $\mathrm{PMH}$ for $\sim 6$ generations at $37^{\circ} \mathrm{C}$, were incubated for $1 \mathrm{~h}$ with ${ }^{35} \mathrm{~S}$-labelled amino acids (DuPont NEN Research Products) as previously described (Fetherston \& Perry, 1994) with or without addition of purified Ybt. Labelled proteins were resolved by SDS-PAGE. Equal amounts of trichloroacetic-acid-precipitable counts were loaded in each lane. Dried gels were exposed to Kodak BioMax MR film at room temperature.

Cells carrying either reporter plasmid pEUPP1 or pEUYbtP were cultivated at $37^{\circ} \mathrm{C}$ for $\sim 6$ generations in $\mathrm{PMH}$ in the presence or absence of $10 \mu \mathrm{M}$ iron. The $\beta$-galactosidase 
Table 1. Bacterial strains and plasmids used

\begin{tabular}{|c|c|c|}
\hline $\begin{array}{l}\text { Strain or } \\
\text { plasmid }\end{array}$ & Relevant characteristics* & $\begin{array}{l}\text { Reference }(s) \text { or } \\
\text { source }\end{array}$ \\
\hline \multicolumn{3}{|l|}{ Y. pestis } \\
\hline $\mathrm{KIM} 6+$ & $\mathrm{Hms}^{+} \mathrm{Ybt}^{+} \mathrm{Lcr}^{-}$ & Fetherston et al. (1995) \\
\hline KIM6-2045.1 & $\mathrm{Hms}^{+} \mathrm{Ybt}^{-}(\Delta p s n 2045.1) \mathrm{Lcr}^{-}$ & Fetherston et al. (1995) \\
\hline KIM6-2045.6 & $\mathrm{Hms}^{+} \mathrm{Ybt}^{-}(\Delta p s n:: k a n 2045.6) \mathrm{Km}^{\mathrm{r}} \mathrm{Lcr}^{-}$ & Fetherston et al. (1996) \\
\hline KIM6-2046.1 & $\mathrm{Hms}^{+} \mathrm{Ybt}^{-}\left(\right.$irp 2::kan2046.1) $\mathrm{Km}^{\mathrm{r}} \mathrm{Lcr}^{-}$ & Fetherston et al. (1995) \\
\hline \multicolumn{3}{|c|}{ Y. enterocolitica } \\
\hline WA-LOX & $\mathrm{Ybt}^{+} \mathrm{Lcr}^{-}$serotype O $: 8$ & $\begin{array}{l}\text { Fetherston et al. (1992); Perry } \\
\text { \& Brubaker (1983) }\end{array}$ \\
\hline \multicolumn{3}{|c|}{ Y.pseudotuberculosis } \\
\hline $\mathrm{PB} 1 /+$ & $\mathrm{Ybt}^{+} \mathrm{Lcr}^{+}$serotype 1 & Perry \& Brubaker $(1983)$ \\
\hline $\mathrm{PB} 1 / 0$ & $\mathrm{Ybt}^{+} \mathrm{Lcr}^{-}$serotype 1 & $\begin{array}{l}\text { Fetherston et al. (1992); Perry } \\
\text { \& Brubaker (1983) }\end{array}$ \\
\hline $\mathrm{PB} 1-2045 /+$ & $p s n::$ pPSN15 $\mathrm{Lcr}^{+} \mathrm{Ap}^{\mathrm{r}}$ Sucs type 1 & This study \\
\hline PB1-2045.1/ + & $\mathrm{Ybt}^{-}(\Delta p s n 2045.1) \mathrm{Lcr}^{+} \mathrm{Ap}^{\mathrm{s}} \mathrm{Suc}^{\mathrm{r}}$ type 1 & This study \\
\hline PB1-2045.1/0 & $\mathrm{Ybt}^{-}(\Delta p s n 2045.1) \mathrm{Lcr}^{-} \mathrm{Ap}^{\text {s }}$ Suc ${ }^{\mathrm{r}}$ type 1 & This study \\
\hline $\mathrm{PB} 1-2046 /+$ & $\operatorname{irp2::pCIRP1} \mathrm{Lcr}^{+} \mathrm{Ap}^{r} \mathrm{Km}^{\mathrm{r}}$ Suc $c^{\mathrm{s}}$ type 1 & This study \\
\hline PB1-2046.1/ + & $\mathrm{Ybt}^{-}\left(\right.$irp 2:: kan2046.1) $\mathrm{Lcr}^{+} \mathrm{Ap}^{\mathrm{s}} \mathrm{Km}^{\mathrm{r}} \mathrm{Suc}^{\mathrm{r}}$ type 1 & This study \\
\hline PB1-2046.1/0 & $\mathrm{Ybt}^{-}$(irp2::kan2046.1) $\mathrm{Lcr}^{-} \mathrm{Ap}^{\mathrm{s}} \mathrm{Km}^{\mathrm{r}}$ Suc ${ }^{\mathrm{r}}$ type 1 & This study \\
\hline \multicolumn{3}{|l|}{ Plasmids } \\
\hline pCIRP498.8 & $\operatorname{irp} 2:: \operatorname{kan} 2046.1$ suicide vector, $\mathrm{Ap}^{\mathrm{r}} \mathrm{Km}^{\mathrm{r}} \mathrm{Suc} \mathrm{c}^{\mathrm{s}}$ & Bearden et al. (1997) \\
\hline pEUPP1 & $\begin{array}{l}\text { Low-copy-number } p s n: \text { : lac } Z \text { reporter plasmid, } \\
\mathrm{Spc}^{\mathrm{r}} \text {; iron-, Fur - and YbtA-regulated expression } \\
\text { of } \beta \text {-galactosidase }\end{array}$ & Fetherston et al. (1996) \\
\hline pEUYbtP & $\begin{array}{l}\text { Low-copy-number ybtP: : lac Z reporter plasmid, } \\
\mathrm{Spc}^{\mathrm{r}} \text {; iron-, Fur - and YbtA-regulated expression } \\
\text { of } \beta \text {-galactosidase }\end{array}$ & Fetherston et al. (1999) \\
\hline pPSN 15 & $\Delta p s n 2045.1$ suicide vector, $\mathrm{Ap}^{\mathrm{r}} \mathrm{Suc}^{\mathrm{s}}$ & Fetherston et al. (1995) \\
\hline
\end{tabular}

* $\mathrm{Ybt}^{+}$, a completely functional yersiniabactin biosynthesis and transport system. $\mathrm{Lcr}^{+} / \mathrm{Lcr}^{-}$, presence or absence, respectively, of the low-calcium-response virulence plasmid. $\mathrm{Hms}^{+}$, functional haeminstorage phenotype. $\mathrm{Ap}^{\mathrm{r}}, \mathrm{Km}^{\mathrm{r}}, \mathrm{Spc}^{\mathrm{r}}, \mathrm{Suc}^{\mathrm{r}}$, resistant to ampicillin, kanamycin, spectinomycin and sucrose, respectively; Suc ${ }^{\mathrm{s}}$, sensitive to sucrose.

activities of cultures were determined spectrophotometrically by following cleavage of 4-nitrophenyl $\beta$-D-galactopy ranoside. $\beta$-Galactosidase activities are expressed in Miller units (Miller, 1992).

A plate assay system was used to determine sensitivity or resistance of $Y$. pseudotuberculosis strains to the yersinial bacteriocin pesticin. From overnight liquid heart infusion broth cultures, $\sim 5 \times 10^{5}$ cells were inoculated into $5 \mathrm{ml}$ molten TBA containing $2.5 \mathrm{mM} \mathrm{CaCl}$ and $2.5 \mathrm{mM}$ EDTA and overlayered onto similarly supplemented TBA plates. Wells of $\sim 1 \mathrm{~mm}$ were formed in seeded plates and $20 \mu \mathrm{l}$ of a 1:50 dilution of partially purified pesticin was added; plates were then incubated for $10-18 \mathrm{~h}$ at $37^{\circ} \mathrm{C}$.

Iron transport studies. $Y$. pestis strains were cultivated at $37^{\circ} \mathrm{C}$ in $\mathrm{PMH}$ for $\sim 6$ generations under iron-deficient conditions. For KIM6-2046.1, purified Ybt was added to yield a 1:16 titre $1 \mathrm{~h}$ prior to the start of transport assays. For energy-poisoned cell controls, the protonophore carbonyl cyanide $m$-chlorophenylhydrazone (CCCP) was added to a final concentration of $100 \mu \mathrm{M} 10 \mathrm{~min}$ prior to the start of transport assays. Assays were initiated in cultures at an $\mathrm{OD}_{620}$ of $\sim 0.4$ by the addition of ${ }^{55} \mathrm{FeCl}_{3}$ to a concentration of $0.2 \mu \mathrm{Ci}(7.4 \mathrm{kBq})$ per $\mathrm{ml}$ of culture. For transport assays with
KIM6-2046.1, additional purified Ybt (to a titre of $1: 32$ ) was added with the radioisotope. Aliquots of $0.5 \mathrm{ml}$ were collected through $0.45 \mu \mathrm{m}$ membrane filters (Gelman Sciences) at regular intervals and washed twice with $\mathrm{PMH}$. Filters were presoaked in $\mathrm{PMH}$ containing $20 \mu \mathrm{M} \mathrm{FeCl}$ to prevent nonspecific adsorption. Direct samples were taken to calculate the percentage uptake of radioisotope. All samples were counted in Bio-Safe II scintillation cocktail (Research Products International) on a Beckman LS3801 liquid scintillation counter using a $0-1000$ window. Results are presented as percentage uptake of isotope per $0.4 \mathrm{OD}_{620}$ (corresponding to $\sim 2.8 \times 10^{7}$ c.f.u. $\mathrm{ml}^{-1}$ ). Control experiments using uninoculated $\mathrm{PMH}$ showed that radioisotope was not retained by the filters (data not shown).

Purification of yersiniabactin and determination of affinity for ferric iron. Isolation of Ybt was achieved using methods modified from Chambers et al. (1996) and Drechsel et al. (1995). In a typical purification procedure Ybt was isolated from 3 litres of filtered supernatant from a $Y$. pestis KIM62045.1 culture grown at $37^{\circ} \mathrm{C}$ in deferrated $\mathrm{PMH}$ for $\sim 8$ generations. Potassium phosphate was added to a concentration of $\sim 1 \mathrm{M}$ at $\mathrm{pH} 7 \cdot 0$ and the solution was extracted twice with 0.5 vol. ethyl acetate. Ethyl acetate fractions were 
pooled, evaporated to near dryness, then brought up to $20 \mathrm{ml}$ in $70 \%$ ethanol. Two C-18 SEP-PAK cartridges were used in a preliminary purification step; the sample was applied, the column washed with $70 \%$ ethanol, and Ybt was eluted with $100 \%$ methanol. Methanol fractions were reduced in volume by evaporation to a volume of $1.6 \mathrm{ml}$. Water and ammonium formate, $\mathrm{pH} \mathrm{8.0,} \mathrm{were} \mathrm{added} \mathrm{to} \mathrm{adjust} \mathrm{to} 10 \mathrm{mM}$ ammonium formate in $\sim 5 \mathrm{ml}$. This was applied to a preparative C-18 HPLC column and eluted with a gradient of $20 \%$ methanol, $10 \mathrm{mM}$ ammonium formate, $\mathrm{pH} 8.0$, to $100 \%$ methanol. Ybt eluted with the $100 \%$ methanol phase, and was detected by its absorbance maximum at $385 \mathrm{~nm}$ (Drechsel et al., 1995) and by bioassay.

$\mathrm{Fe}^{3+}$-saturated $\mathrm{Ybt}$ and EDTA were used in competition experiments to determine the formation constant for Ybt with ferric iron. Competition reactions containing $16 \mu \mathrm{M} \mathrm{Ybt- \textrm {Fe } ^ { 3 + }}$ in a final volume of $1 \mathrm{ml}$ were reacted with ten concentrations of EDTA over a range from 0 to $63.0 \mathrm{mM}$ at a $\mathrm{pH}$ of 8.35 . All reactions were allowed to reach equilibrium by incubating for $24 \mathrm{~h}$ at $37^{\circ} \mathrm{C}$ and then were stored at $-70^{\circ} \mathrm{C}$ until analysis. Competition reactions were analysed by HPLC employing an analytical C-18 column, and the final $\mathrm{Ybt}-\mathrm{Fe}^{3+}$ concentrations for each reaction were calculated from $A_{385}$ values.

Ybt bioassay. Y. pestis KIM6-2046.1 cells were cultured and overlayered onto PMH-S plates as previously described (Fetherston et al., 1995). The irp2: : kan2046.1 mutation in this strain prevents $\mathrm{Ybt}$ synthesis; consequently it cannot grow on $\mathrm{PMH}-\mathrm{S}$ plates at $37^{\circ} \mathrm{C}$ unless supplied with an exogenous source of Ybt (Fetherston et al., 1995). Fractions from Ybt purification procedures or filtered culture supernatants from Y. pestis, Y. pseudotuberculosis and Y. enterocolitica strains were added to wells bored into the agar to test for growth simulation of KIM6-2046.1 at $37^{\circ} \mathrm{C}$.

\section{RESULTS}

\section{Isolation and characterization of yersiniabactin}

Ybt from Y. pestis KIM6-2045.1 was isolated as described in Methods. HPLC UV/visible diode array analysis indicated that the preparation of $\mathrm{Ybt}$ was free of detectable contaminants. Bioassays demonstrated that the purified Ybt preparation possessed growthstimulatory activity for Y. pestis KIM6-2046.1 at a dilution of 1:64000 but did not support the growth of KIM6-2045.1, which lacks the Ybt OM receptor, on PMH-S plates. Supernatants from iron-deficient cultures of KIM $6+$ after $\sim 8$ generations of growth generally possess growth-stimulatory activity at dilutions of $1: 8$ or $1: 16$.

The iron-saturated form of $\mathrm{Ybt}$ was analysed by UV/visible spectroscopy and found to produce a spectrum at $\mathrm{pH} 7$ indistinguishable from that published by Haag et al. (1993) and Chambers et al. (1996), with absorption maxima at approximately 255,310 and $385 \mathrm{~nm}$ (data not shown). The high-resolution matrixassisted laser desorption (MALDI) mass analysis (positive ion mode) of iron-saturated Ybt yielded a spectrum with major ion intensities at $\mathrm{m} / z 295.056,489.029$, 535.035 and 572.992 (Fig. 2). The 535.035 and 572.992 ions are consistent with the formula $\mathrm{C}_{21} \mathrm{H}_{24} \mathrm{~N}_{3} \mathrm{O}_{4} \mathrm{~S}_{3} \mathrm{HFe}^{+}$ (calculated 535.036) and $\mathrm{C}_{21} \mathrm{H}_{24} \mathrm{~N}_{3} \mathrm{O}_{4} \mathrm{~S}_{3} \mathrm{KFe}^{+}$(calculated 572.992), respectively, and represent the $\mathrm{FeH}^{+}$and $\mathrm{FeK}^{+}$ adducts of the $\mathrm{Ybt}$ molecule also seen in the purification

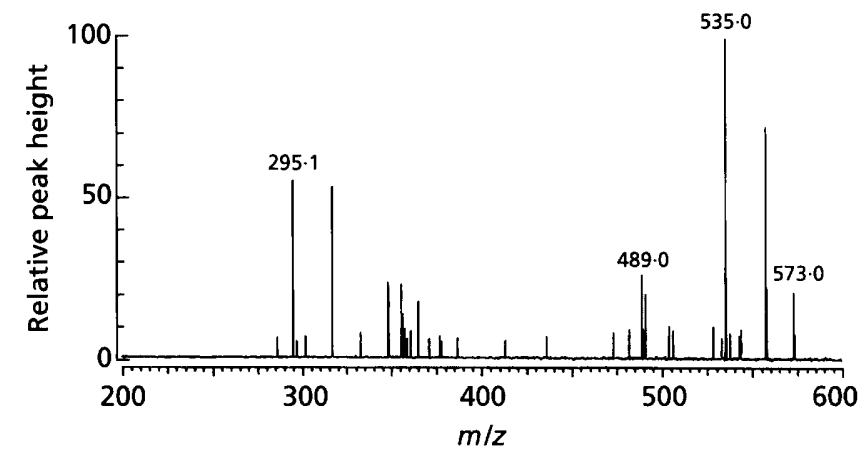

Fig. 2. MALDI mass spectrum of Fe-yersiniabactin isolated from Y. pestis.

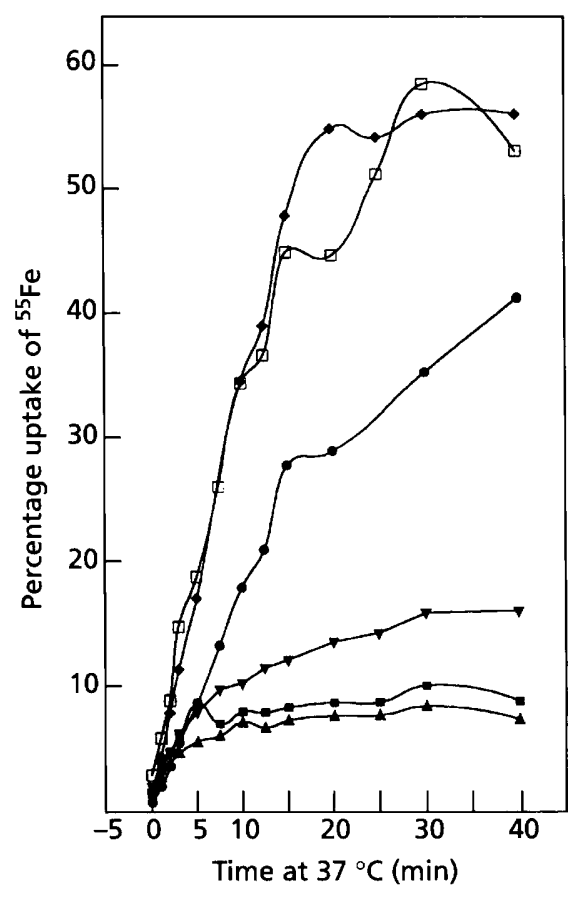

Fig. 3. Uptake of iron by $Y$. pestis KIM cells. $\square, \boldsymbol{\square}, \mathrm{Ybt}^{+} \mathrm{KIM} 6+$ without $(\square)$ and with $100 \mu \mathrm{M}$ CCCP $(\square) ; \nabla, \Delta$, Psn $^{-}$KIM62045.6 without $(\nabla)$ and with $100 \mu \mathrm{M} \mathrm{CCCP}(\Delta) ; 0, \diamond, \mathrm{Ybt}^{-}$ KIM6-2046.1 without (O) and with exogenous Ybt siderophore $(\bullet)$ (both without CCCP). Iron uptake is presented as percentage uptake per $0.4 O_{620}$ of cells to normalize for differences between cultures and for bacterial growth during the assay period.

of Ybt from Y. enterocolitica (Chambers et al., 1996; Drechsel et al., 1995). The $m / z 295$ ion was also seen in mass spectra of the siderophore from Y. enterocolitica and the Ybt cleavage product it represents was identified (Drechsel et al., 1995); however, the 489 ion was not detected. This is possibly due to our use of MALDI rather than an electrospray interface. The 489 ion probably arises from decarboxylation of the molecular ion of the $\mathrm{FeH}^{+}$adduct. Decarboxylation of the $\mathrm{Al}^{3+}$ adduct of $\mathrm{Ybt}$ from Y. enterocolitica was observed in high-resolution electron-impact mass spectrometry 
Table 2. $\beta$-Galactosidase activities of $Y$. pestis and $Y$. pseudotuberculosis strains grown at $37^{\circ} \mathrm{C}$ to mid-exponential phase in $\mathrm{PMH}$ medium

\begin{tabular}{|c|c|c|c|c|}
\hline \multirow[t]{2}{*}{ Strain } & \multicolumn{4}{|c|}{$\beta$-Galactosidase activity* of cells grown in PMH with: } \\
\hline & $10 \mu \mathrm{M} \mathrm{FeCl}_{3}$ & $\begin{array}{c}\text { No added iron } \\
\text { or Ybt }\end{array}$ & Ybt $(1: 16$ titre $)$ & $\begin{array}{l}\text { Minus/plus } \\
\text { ratio } \dagger\end{array}$ \\
\hline \multicolumn{5}{|l|}{ Y.pestis } \\
\hline KIM6(pEUPP1)+ & $\mathrm{ND}$ & $2035( \pm 467)$ & ND & ND \\
\hline $\begin{array}{l}\text { KIM6-2046.1 } \\
\text { (pEUPP1) }\end{array}$ & ND & $616( \pm 372)$ & $3806( \pm 697)$ & $5 \cdot 9$ \\
\hline KIM6(pEUYbtP) + & $500( \pm 207)$ & $28606( \pm 11615)$ & ND & 57 \\
\hline KIM6-2046.1(pEUYbtP) & $141( \pm 13)$ & $1787( \pm 351)$ & ND & 13 \\
\hline \multicolumn{5}{|l|}{ Y. pseudotuberculosis } \\
\hline PB1/0(pEUYbtP) & $84( \pm 23)$ & $13629( \pm 4318)$ & $\mathrm{ND}$ & 162 \\
\hline PB1-2046.1/0(pEUYbtP) & $73( \pm 13)$ & $505( \pm 112)$ & $\mathrm{ND}$ & 7 \\
\hline
\end{tabular}

*Activity is expressed in Miller units; the data are means from two to five experiments, with standard deviations in parentheses. ND, Not determined.

$\dagger-\mathrm{Fe} /+$ Fe ratio for all strains except KIM6-2046.1(pEUPP1) $(-\mathrm{Ybt} /+\mathrm{Ybt}$ ratio).

Table 3. Growth of $Y$. pestis and $Y$. pseudotuberculosis derivatives on PMH-S at $37{ }^{\circ} \mathrm{C}$

\begin{tabular}{|c|c|c|c|}
\hline Strain & Growth on PMH-S & $\begin{array}{l}\text { Cross-feeding } \\
\text { of KIM6-2046.1 } \\
\text { on } \\
\text { PMH-S }\end{array}$ & $\begin{array}{c}\text { Cross-fed by } \\
\text { KIM6-2045.1 or PB1/0 } \\
\text { on PMH-S-75 } \mu \mathrm{M} \\
\text { DIP }\end{array}$ \\
\hline \multicolumn{4}{|l|}{$Y$. pestis } \\
\hline $\mathrm{KIM} 6+\left(\mathrm{Psn}^{+} \mathrm{Ybt}^{+}\right)$ & + & + & ND \\
\hline $\begin{array}{l}\text { KIM6-2046.1 } \\
\text { (irp2::kan2046.1) }\end{array}$ & - & - & $\mathrm{ND}$ \\
\hline KIM6-2045.1 ( $\Delta p s n 2045.1)$ & - & + & ND \\
\hline \multicolumn{4}{|l|}{ Y. pseudotuberculosis } \\
\hline $\mathrm{PB} 1 / 0\left(\mathrm{Psn}^{+} \mathrm{Ybt}^{+}\right)$ & + & + & ND \\
\hline $\begin{array}{l}\text { PB1-2046.1/0 } \\
\quad(\text { irp2: : kan2046.1) }\end{array}$ & - & - & + \\
\hline $\begin{array}{l}\mathrm{PB} 1-2045.1 / 0 \\
(\Delta p \operatorname{sn} 2045.1)\end{array}$ & - & - & - \\
\hline
\end{tabular}

ND, Not determined.

(Chambers et al., 1996). Thus Ybt synthesized by Y. pestis appears to be identical to the siderophore synthesized by Y. enterocolitica (Chambers et al., 1996; Drechsel et al., 1995). The mass spectrum also supports the octahedral coordination of the ferric iron atom by a phenolate, three sets of electron pairs from the nitrogen atoms, an electron pair from the oxygen of the nonionized secondary alcohol, and a carboxylate (see Fig. 1).

\section{Formation constant for $\mathrm{Fe}^{3+}$-yersiniabactin}

Titration of Ybt in methanol with $0.5 \mathrm{M} \mathrm{FeCl}_{3}$ reduced the $A_{385}$. In contrast, titration of purified Ybt with $\mathrm{FeSO}_{4}$ under strictly anaerobic conditions did not alter the Ybt spectrum (data not shown), indicating that $\mathrm{Ybt}$ binds $\mathrm{Fe}^{3+}$ with a much greater affinity than $\mathrm{Fe}^{2+}$. Ybt was saturated with iron by titrating a small volume in methanol with $0.5 \mathrm{M} \mathrm{FeCl}_{3}$ until the $A_{385}$ reached a minimum value. The $\mathrm{Fe}^{3+}$-saturated $\mathrm{Ybt}$ preparation was used in competition reactions with EDTA to determine a formation constant for $\mathrm{Ybt}$ with $\mathrm{Fe}^{3+}$.

A proton-independent formation constant $\left(K_{\mathrm{Ybt}}^{\mathrm{PI}}\right)$ for Ybt with $\mathrm{Fe}^{3+}$ was determined from the observed formation constant $\left(K_{\mathrm{obs}, \mathrm{Ybt}}\right)$ and $a_{\mathrm{H} 1}$, the fraction of Ybt calculated to be in the singly protonated form. The values of the acid dissociation constants $\left(\mathrm{p} K_{1}\right.$ to $\left.\mathrm{p} K_{6}\right)$ for Ybt were approximated from published values for compounds representing the various functional groups. 


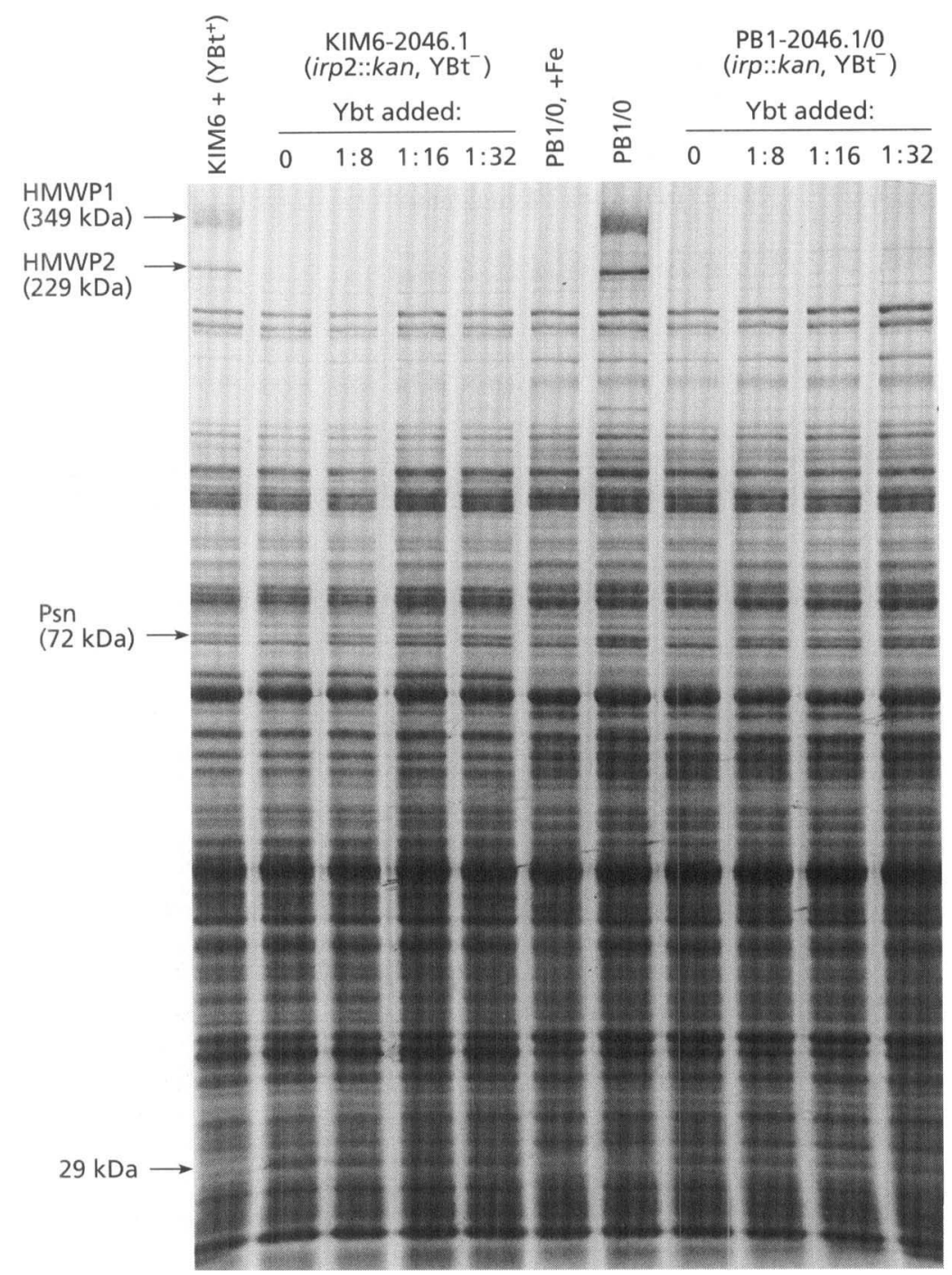

Fig. 4. SDS-PAGE analysis of whole-cell proteins from $Y$. pestis and $Y$. pseudotuberculosis cells grown in PMH with or without added $\mathrm{FeCl}_{3}(10 \mu \mathrm{M})$ or $\mathrm{Ybt}$ siderophore. Concentrations of Ybt added are given as growth-response titres (i.e. 1:32 is the highest concentration of added $\mathrm{Ybt}$ ). Molecular masses of HMWP1, HMWP2 and Psn are from the deduced amino acid sequence and not estimates based on migration in SDS-PAGE.

These were $\mathrm{p} K_{1}=3 \cdot 10$ (aliphatic-substituted thiazoline) ; $\mathrm{pK}_{2}=3.12$ (phenyl-substituted thiazoline) (Schmir, $1965) ; \mathrm{pK}_{3}=4.80$ (carboxylic acid) $; \mathrm{p} K_{4}=9.89$ (phenol) (Bird \& Cheeseman, 1984); $\quad \mathrm{p} K_{5}=11.3$ (thiazolidine) (Schmir, 1965); and $\mathrm{p} K_{6}=16$ (secondary alcohol) (Bordwell, 1963), which was assumed to be protonated in the calculation. A similar calculation was performed for EDTA using $\mathrm{p} K$ values of $2 \cdot 0,2 \cdot 7,6 \cdot 2$ and 10.3 (Dawson et al., 1987). Using these values, the proton-independent formation constant of $\mathrm{Fe}^{3+}-\mathrm{Ybt}$ $\left(K_{\mathrm{Ybt}}^{\mathrm{PI}}\right)$ was determined to be $36 \cdot 6$ (or $\sim 4 \times 10^{-36}$ ).

\section{Yersiniabactin enhances iron uptake}

Fig. 3 shows uptake of iron by iron-starved Y.pestis cells possessing all the genes needed for $\mathrm{Ybt}$ synthesis and utilization (KIM6 +) or with a mutation in either a Ybt biosynthetic operon (KIM6-2046.1, irp2:: kan2046.1) or the OM receptor for Ybt (KIM6-2045.6, $\Delta p s n:: k a n 2045.6)$. Iron uptake by KIM6 + cells was energy-dependent and essentially complete after 15-20 min. Energy-independent binding of iron to KIM6 +, KIM6-2045.6 and KIM6-2046.1 cells was nearly identical (Fig. 3 and data not shown). Energy-dependent uptake of iron by KIM6-2046.1 cells was significantly reduced relative to KIM6 + cells, while KIM6-2045.6 cells did not exhibit substantial energy-dependent uptake of iron. Addition of purified Ybt to KIM6-2046.1 cells stimulated iron uptake to levels similar to that observed in $\mathrm{Ybt}^{+}$ KIM6 + cells (Fig. 3).

\section{Role of yersiniabactin in gene regulation}

Previously we showed that a mutant of $Y$. pestis unable to syntheize Ybt (KIM6-2046.1) was defective in expression of Psn, the OM receptor for Ybt. Expression levels of Psn were enhanced when cells were incubated with filtered culture supernatant from a Ybt-producing Y. pestis strain (Fetherston et al., 1995). To clearly demonstrate that the supernatant component responsible for this enhanced expression was Ybt, we performed similar experiments using purified Ybt. Fig. 4 


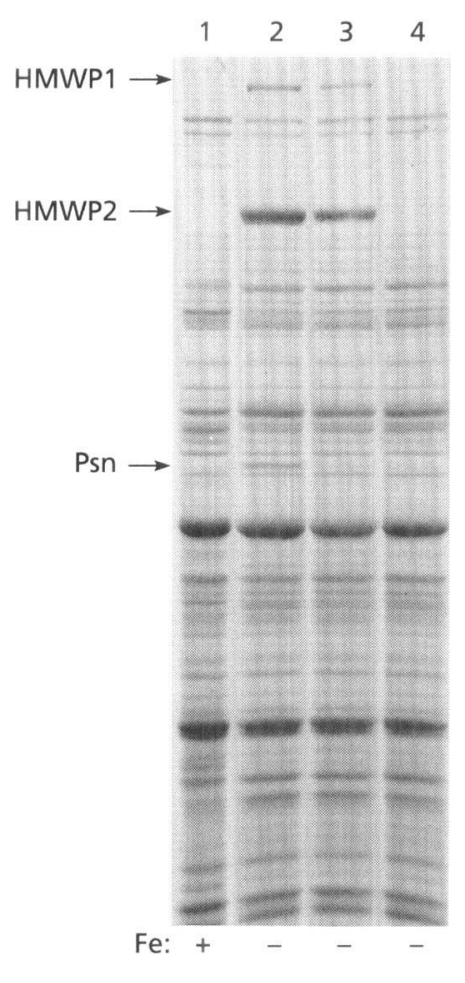

Fig. 5. SDS-PAGE analysis of whole-cell proteins from $Y$. pseudotuberculosis cells grown in PMH with $10 \mu \mathrm{M} \mathrm{FeCl}_{3}$ (lane 1) or without added iron (lanes 2-4). Lanes 1 and $2, Y$. pseudotuberculosis PB 1/0 (pEUYbtP); lane 3, Y. pseudotuberculosis PB-2045.1/0(pEUYbtP)( $\Delta$ psn2045.1); lane 4, Y. pseudotuberculosis PB-2046.1/0(pEUYbtP) (irp2::kan2046.1).

shows that expression of Psn by KIM6-2046.1 cells is enhanced by adding increasing concentrations of $\mathrm{Ybt}$ to the culture $1 \mathrm{~h}$ prior to harvesting cells for SDS-PAGE analysis. HMWP1, HMWP2, YbtE and other Ybt proteins encoded within this operon are not expressed in this mutant due to polar effects of the irp2::kan insertion. Addition of purified $\mathrm{Ybt}$ also enhanced expression of an $\sim 29 \mathrm{kDa}$ polypeptide which was not observed in iron-deficient cells of KIM6+. Within the high-pathogenicity island that encodes the Ybt system, only $\mathrm{YbtA}$ has a corresponding molecular mass (Fetherston et al., 1996; Gehring et al., 1998a). If this band does correspond to $\mathrm{YbtA}$, the experimental conditions used may have caused an abnormally high level of expression of this regulatory protein.

We used a well-characterized reporter to demonstrate that Ybt exerts its regulatory effects at the level of gene transcription. Reporter plasmid pEUPP1 is a low-copynumber plasmid in which expression of lac $Z$ is driven by the $p s n$ promoter ( $p s n:: l a c Z$ ) (Table 1). This construct was previously used to show that transcription from this promoter is iron and Fur regulated and requires $\mathrm{YbtA}$ for maximal expression (Fetherston et al., 1996). Table 2 shows that $Y$. pestis cells unable to synthesize $\mathrm{Ybt}$ (KIM6-2046.1) reduced transcriptional activity from the psn promoter by a factor of three when compared to cells synthesizing Ybt (KIM6+). Addition of purified Ybt $1 \mathrm{~h}$ prior to collecting assay samples caused a sixfold increase in transcriptional activity.

\section{The yersiniabactin system and Y. pseudotuberculosis}

Although there is now abundant evidence for the identity of the Ybt systems in $Y$. pestis and Y. enterocolitica (Bearden et al., 1997; Fetherston et al., 1995, 1996; Gehring et al., 1998a ; Guilvout et al., 1993; Pelludat et al., 1998), studies using Y. pseudotuberculosis are rare. Consequently, we constructed $\Delta p s n 2045.1$ and irp2::kan2046.1 mutations in Y. pseudotuberculosis. As expected, Y. pseudotuberculosis PB1-2045.1/0 ( $\Delta p s n 2045.1)$ cells were resistant to the bacteriocin pesticin, which uses the Ybt receptor. In addition, neither Y. pseudotuberculosis mutant was able to grow on PMH-S plates at $37^{\circ} \mathrm{C}$ (Table 3), while the PB1/0 parental strain was able to grow at $37^{\circ} \mathrm{C}$ on PMH-S containing $75 \mu \mathrm{M}$ DIP. Similar results were obtained with parental and mutant strains of $Y$. pestis (Table 3 and data not shown).

We have previously shown that culture supernatants from Y. pestis KIM6-2045.1 ( $\Delta p s n 2045.1$ ) but not KIM62046.1 (irp:: kan2046.1) cells grown under iron-deficient conditions can stimulate growth of KIM6-2046.1 cells on PMH-S plates (Fetherston et al., 1995). While culture supernatants from $Y$. pseudotuberculosis $\mathrm{PB} 1 / 0$ parental cells stimulated the growth of Y. pestis KIM62046.1 on PMH-S plates, supernatants from both Y. pseudotuberculosis mutants failed to provide growthpromoting activity (Table 3 ). Supernatants from irondeficient cultures of $Y$. pestis KIM6-2045.1 and Y. pseudotuberculosis PB1/0 stimulated growth of $Y$. pseudotuberculosis PB1-2046.1/0 but not PB1-2045.1/0 cells on PMH-S-75 $\mu$ M DIP plates at $37^{\circ} \mathrm{C}$ (Table 3). These results suggest that both $Y$. pseudotuberculosis mutants are unable to synthesize or secrete significant quantities of Ybt siderophore under iron-starvation conditions, and that only the Irp $2^{-}$mutant is able to use exogenous $\mathrm{Ybt}$ siderophore for growth.

Fig. 4 shows that $Y b t$ is also necessary for maximal expression of the Ybt OM receptor, Psn and the unidentified $\sim 29 \mathrm{kDa}$ polypeptide in Y. pseudotuberculosis PB1-2046.1 (irp2::kan2046). These results are essentially identical to those obtained with the same mutation in $Y$. pestis (Fig. 4). We also analysed transcription from a Ybt-regulated promoter fused to lacZ in the Y. pseudotuberculosis strains (Table 2). For this study we used a low-copy-number vector carrying lacZ fused to the $y b t P$ promoter. $\mathrm{YbtP}$ is a cytoplasmic membrane permease required for transport of iron from Ybt into the cell. Like $p s n$, transcription of the $y b t P$ promoter in Y. pestis is iron, Fur and YbtA regulated and requires Ybt for maximum expression (Table 2; Fetherston et al., 1998). The results indicate that transcription from this promoter in Y. pseudotuberculosis is tightly iron regulated. A mutation in the irp2 gene completely repressed transcription (Table 2). 
Although the $Y$. pseudotuberculosis strain carrying the $\Delta p s n$ mutation showed slightly reduced levels of HMWP1 and HMWP2 expression (Fig. 5), $\beta$ galactosidase expression from pEUYbtP in this strain was inconclusive and statistically unreliable (data not shown).

\section{DISCUSSION}

Given the high degree of sequence identities between the genes encoding the $Y$. pestis and Y. enterocolitica $\mathrm{Ybt}$ systems (Bearden et al., 1997; Fetherston et al., 1995, 1996; Gehring et al., 1998a; Guilvout et al., 1993; Pelludat et al., 1998), we have assumed that the siderophores from these organisms would have identical or nearly identical structures. The UV/visible spectrum and mass analysis of $\mathrm{Ybt}$ from $Y$. pestis indicates that it is indeed identical to the siderophore produced by $Y$. enterocolitica. Like many other siderophores, Ybt has little or no affinity for binding ferrous iron but has a formation constant of $4 \times 10^{-36}$ for ferric iron. Thus Ybt has a higher affinity for ferric iron than a number of other siderophores such as ferrichrome, the ferrioxamines, aerobactin and pyoverdin (Albrecht-Gary \& Crumbliss, 1998). It would be interesting to compare the binding constant of $\mathrm{Ybt}$ with pyochelin and anguibactin, siderophores produced by $P$. aeruginosa and $V$. anguillarum, respectively, that are structurally similar to Ybt (Fig. 1). Unfortunately, the affinities of pyochelin and anguibactin for iron apparently have not been experimentally determined.

As in Y.enterocolitica (Haag et al., 1993), Ybt functions to transport iron into Y. pestis (Fig. 3). Thus KIM62046.1, a strain unable to produce $\mathrm{Ybt}$, accumulates iron at reduced levels relative to KIM6 +, which possesses an intact Ybt system. This residual iron transport in KIM62046.1 is presumably due to the presence of other lowaffinity iron-transport systems in Y. pestis, such as the Yfe system (Bearden et al., 1998). Addition of purified Ybt to KIM6-2046.1 cells restored iron transport to wild-type levels seen with KIM6 + cells. Iron uptake by KIM6-2045.6, a strain bearing a mutation in the OM receptor for Ybt, was substantially lower than for KIM62046.1. KIM6-2045.6 cells still synthesize and secrete $\mathrm{Ybt}$, which then presumably binds iron in the medium, making it unavailable for transport by other irontransport systems.

Ybt apparently plays a regulatory role in controlling its own synthesis as well as expression of the Ybt OM receptor, Psn. We previously reported that cell-free culture supernatants containing $\mathrm{Ybt}$ enhanced expression of HMWP1, HMWP2 and YbtE, proteins involved in Ybt biosynthesis (Gehring et al., 1998a, b) as well as Psn. In addition, cells unable to synthesize Ybt show reduced levels of transcription from the $p s n$ and ybtP promoters (Bearden et al., 1997; Fetherston et al., 1995, 1999). Using Ybt purified from Y. enterocolitica, Pelludat et al. (1998) subsequently demonstrated regulation of Y. enterocolitica HMWP2 and Psn proteins. We have confirmed and extended our findings using purified Ybt. Addition of Ybt to iron-deficient cells of KIM6-2046.1 greatly increased expression of Psn (Fig. 4). Using a transcriptional reporter, we demonstrated that this regulation occurs at the level of transcription (Table 2).

The Ybt systems present in the three pathogenic Yersinia spp. are closely related and functionally interchangeable. DNA sequence similarities (Rakin et al., 1995) and the ease of allelic exchange of a mutated Y. enterocolitica irp2 gene into Y. pseudotuberculosis (Carniel et al., 1992) suggest that the $Y$. pseudotuberculosis Ybt genes are nearly identical to those of $Y$. enterocolitica. We have extended these findings by showing that $Y$. pseudotuberculosis culture supernatants containing $\mathrm{Ybt}$ support the growth of $Y$. pestis and that mutated $Y$. pestis psn and irp2 genes can be used to construct $Y$. pseudotuberculosis mutants. The Y. pseudotuberculosis irp2 mutant failed to synthesize Ybt, while the $p s n$ mutant was unable to use Ybt for growth. In addition, Ybt also serves a regulatory role in Y. pseudotuberculosis. A mutant unable to synthesize Ybt had no detectable expression of Psn and partially repressed transcription from the $y b t P$ promoter. Exogenous $\mathrm{Ybt}$ restored expression of the Ybt OM receptor Psn to this mutant.

Regulation of expression by small molecules involved in iron transport is not unprecedented; for example ferric citrate in E. coli and several of the siderophores produced by Pseudomonas species induce expression of their respective receptors (Braun et al., 1998; Heinrichs $\&$ Poole, 1996; Venturi et al., 1995). In most cases, the receptor is also needed for induction. However, deletion of $p s n$ in $Y$. pestis did not reduce expression of a psn::lacZ reporter gene or genes for $\mathrm{Ybt}$ biosynthesis (Bearden et al., 1997; Fetherston et al., 1996, 1999). This is in contrast to recent results with $Y$. enterocolitica, where a Ybt receptor mutant displayed reduced levels of HMWP2, suggesting that the receptor is important for regulation (Pelludat et al., 1998). Although earlier studies from the same laboratory noted increased siderophore synthesis and secretion from the same mutant strain (Rakin et al., 1994), this anomaly has not been addressed. In Y. pseudotuberculosis, a psn mutation resulted in a slight reduction in expression of HMWP1 and HMWP2 (Fig. 5). This strain also did not produce levels of $\mathrm{Ybt}$ siderophore detectable by bioassay. In contrast, the same mutation in psn in $Y$. pestis does not repress expression of HMWP1, HMWP2 or Ybt siderophore (Fetherston et al., 1995).

Thus, identical mutations in Y. pestis and Y. pseudotuberculosis have slightly different regulatory effects. One possible explanation for the different regulatory effects of $\mathrm{Psn}^{-}$mutants in Y. pestis and the enteropathogenic yersiniae would be putative differences in Ybt permeation through the OM. Presumably the OM of $Y$. pestis allows sufficient diffusion of $\mathrm{Ybt}$ into the periplasm to serve as a regulatory signal while the OM of Y. pseudotuberculosis allows reduced diffusion and the Y. enterocolitica OM is essentially impermeable to 
Ybt. In this model Psn is a 'signal transducer' only in the sense that it is translocating Ybt across the OM. It is intriguing that a recent study of the pathogenic yersinias concluded that the OM of Y. pestis was most permeable to small hydrophobic molecules while the $Y$. enterocolitica OM was least permeable to these molecules (Bengoechea et al., 1998).

\section{ACKNOWLEDGEMENTS}

The authors thank Karen Meekins for help with some experiments. They also thank Dr Amy Harms and Dr Jan Pyrek for performing mass spectrometry at the University of Kentucky Mass Spectrometry Facility. This work was supported by National Institutes of Health grant A1042738.

\section{REFERENCES}

Albrecht-Gary, A.-M. \& Crumbliss, A. L. (1998). Coordination chemistry of siderophores: thermodynamics and kinetics of iron chelation and release. In Metal Ions in Biological Systems, pp. 239-327. Edited by A. Sigel \& H. Sigel. New York: Marcel Dekker.

de Almeida, A. M. P., Guiyoule, A., Guilvout, I., Iteman, I., Baranton, G. \& Carniel, E. (1993). Chromosomal irp2 gene in Yersinia: distribution, expression, deletion and impact on virulence. Microb Pathog 14, 9-21.

Bearden, S. W., Fetherston, J. D. \& Perry, R. D. (1997). Genetic organization of the yersiniabactin biosynthetic region and construction of avirulent mutants in Yersinia pestis. Infect Immun 65, 1659-1668

Bearden, S. W., Staggs, T. M. \& Perry, R. D. (1998). An ABC transporter system of Yersinia pestis allows utilization of chelated iron by Escherichia coli SAB11. J Bacteriol 180, 1135-1147.

Bengoechea, J.-A., Brandenburg, K., Seydel, U., Díaz, R. \& Moriyón, I. (1998). Yersinia pseudotuberculosis and Yersinia pestis show increased outer membrane permeability to hydrophobic agents which correlates with lipopolysaccharide acylchain fluidity. Microbiology 144, 1517-1526.

Bird, C. W. \& Cheeseman, G. W. M. (1984). The structure, reactions, synthesis, and uses of heterocyclic compounds. In Comprehensive Heterocyclic Chemistry - 1984. Edited by A. R. Katritzky \& C. W. Rees. Oxford: Pergamon Press.

Birnboim, H. C. \& Doly, J. (1979). A rapid alkaline extraction procedure for screening recombinant plasmid DNA. Nucleic Acids Res 7, 1513-1523.

Bordwell, F. G. (1963). Organic Chemistry. New York: Macmillan.

Braun, V., Hantke, K. \& Köster, W. (1998). Bacterial iron transport: mechanisms, genetics, and regulation. In Metal Ions in Biological Systems, pp. 67-145. Edited by A. Sigel \& H. Sigel. New York: Marcel Dekker.

Buchrieser, C., Prentice, M. \& Carniel, E. (1998). The 102-kilobase unstable region of Yersinia pestis comprises a high-pathogenicity island linked to a pigmentation segment which undergoes internal rearrangement. J Bacteriol 180, 2321-2329.

Byers, B. R. \& Arceneaux, E. L. (1998). Microbial iron transport: iron acquisition by pathogenic microorganisms. In Metal lons in Biological Systems, pp. 37-66. Edited by A. Sigel \& H. Sigel. New York: Marcel Dekker.

Carniel, E., Guiyoule, A., Guilvout, I. \& Mercereau-Puijalon, O. (1992). Molecular cloning, iron-regulation and mutagenesis of the irp2 gene encoding HMWP2, a protein specific for the highly pathogenic Yersinia. Mol Microbiol 6, 379-388.

Carniel, E., Guilvout, I. \& Prentice, M. (1996). Characterization of a large chromosomal 'high-pathogenicity island' in biotype 1B Yersinia enterocolitica. J Bacteriol 178, 6743-6751.

Chambers, C. E. \& Sokol, P. A. (1994). Comparison of siderophore production and utilization in pathogenic and environmental isolates of Yersinia enterocolitica. J Clin Microbiol 32, 32-39.

Chambers, C. E., Mcintyre, D. D., Mouck, M. \& Sokol, P. A. (1996). Physical and structural characterization of yersiniophore, a siderophore produced by clinical isolates of Yersinia enterocolitica. BioMetals 9, 157-167.

Cox, C. D., Rinehart, K. L., Jr, Moore, M. L. \& Cook, C. J., Jr (1981). Pyochelin: novel structure of an iron-chelating growth promoter for Pseudomonas aeruginosa. Proc Natl Acad Sci USA 78, 4256-4260.

Dawson, R. M. C., Elliot, D. C., Elliot, W. H. \& Jones, K. M. (1987). Data for Biochemical Research, 3rd edn. New York: Oxford University Press.

Drechsel, H., Stephan, H., Lotz, R., Haag, H., Zähner, H., Hantke, K. \& Jung, G. (1995). Structure elucidation of yersiniabactin, a siderophore from highly virulent Yersinia strains. Liebigs Ann 1995, 1727-1733.

Fetherston, J. D. \& Perry, R. D. (1994). The pigmentation locus of Yersinia pestis KIM6 + is flanked by an insertion sequence and includes the structural genes for pesticin sensitivity and HMWP2. Mol Microbiol 13, 697-708.

Fetherston, J. D., Schuetze, P. \& Perry, R. D. (1992). Loss of the pigmentation phenotype in Yersinia pestis is due to the spontaneous deletion of $102 \mathrm{~kb}$ of chromosomal DNA which is flanked by a repetitive element. Mol Microbiol 6, 2693-2704.

Fetherston, J. D., Lillard, J. W., Jr \& Perry, R. D. (1995). Analysis of the pesticin receptor from Yersinia pestis: role in iron-deficient growth and possible regulation by its siderophore. J Bacteriol 177, 1824-1833.

Fetherston, J. D., Bearden, S. W. \& Perry, R. D. (1996). YbtA, an AraC-type regulator of the Yersinia pestis pesticin/yersiniabactin receptor. Mol Microbiol 22, 315-325.

Fetherston, J. D., Bertolino, V. J. \& Perry, R. D. (1999). YbtP and YbtQ: two $A B C$ transporter proteins required for iron uptake in Yersinia pestis. Mol Microbiol (in press).

Gehring, A., DeMoll, E., Fetherston, J. D., Mori, I., Mayhew, G. F., Blattner, F. R., Walsh, C. T. \& Perry, R. D. (1998a). Iron acquisition in plague: modular logic in enzymatic biogenesis of yersiniabactin by Yersinia pestis. Chem Biol 5, 573-586.

Gehring, A. M., Mori, I., Perry, R. D. \& Walsh, C. T. (1998b). The nonribosomal peptide synthetase HMWP2 forms a thiazoline ring during biogenesis of yersiniabactin, an iron-chelating virulence factor of Yersinia pestis. Biochemistry 37, 11637-11650.

Guerinot, M. L. (1994). Microbial iron transport. Annu Rev Microbiol 48, 743-772.

Guilvout, I., Mercereau-Puijalon, O., Bonnefoy, S., Pugsley, A. P. \& Carniel, E. (1993). High-molecular-weight protein 2 of Yersinia enterocolitica is homologous to AngR of Vibrio anguillarum and belongs to a family of proteins involved in nonribosomal peptide synthesis. J Bacteriol 175, 5488-5504.

Haag, H., Hantke, K., Drechsel, H., Stojiljkovic, I., Jung, G. \& Zähner, H. (1993). Purification of yersiniabactin: a siderophore and possible virulence factor of Yersinia enterocolitica. J Gen Microbiol 139, 2159-2165.

Heinrichs, D. E. \& Poole, K. (1996). PchR, a regulator of ferripyochelin receptor gene $(f p t A)$ expression in Pseudomonas 
aeruginosa, functions both as an activator and as a repressor. $J$ Bacteriol 178, 2586-2592.

Higuchi, K. \& Smith, J. L. (1961). Studies on the nutrition and physiology of Pasteurella pestis. VI. A differential plating medium for the estimation of the mutation rate to avirulence. J Bacteriol 81, 605-608.

Humphreys, G. O., Willshaw, G. A. \& Anderson, E. S. (1975). A simple method for the preparation of large quantities of pure plasmid DNA. Biochim Biophys Acta 383, 457-463.

Jalal, M. A. F., Hossain, M. B., van der Helm, D., Sanders-Loehr, J., Actis, L. A. \& Crosa, J. H. (1989). Structure of anguibactin, a unique plasmid-related bacterial siderophore from the fish pathogen Vibrio anguillarum. J Am Chem Soc 111, 292-296.

Mietzner, T. A. \& Morse, S. A. (1994). The role of iron-binding proteins in the survival of pathogenic bacteria. Annu Rev Nutr 14, 471-493.

Miller, J. H. (1992). A Short Course in Bacterial Genetics. A Laboratory Manual and Handbook for Escherichia coli and Related Bacteria. Cold Spring Harbor, NY: Cold Spring Harbor Laboratory.

Pelludat, C., Rakin, A., Jacobi, C. A., Schubert, S. \& Heesemann, J. (1998). The yersiniabactin biosynthetic gene cluster of Yersinia enterocolitica: organization and siderophore-dependent regulation. J Bacteriol 180, 538-546.

Perry, R. D. \& Brubaker, R. R. (1983). Vwa ${ }^{+}$phenotype of Yersinia enterocolitica. Infect Immun 40, 166-171.
Rakin, A., Saken, E., Harmsen, D. \& Heesemann, J. (1994). The pesticin receptor of Yersinia enterocolitica: a novel virulence factor with dual function. Mol Microbiol 13, 253-263.

Rakin, A., Urbitsch, P. \& Heesemann, J. (1995). Evidence for two evolutionary lineages of highly pathogenic Yersinia species. $J$ Bacteriol 177, 2292-2298.

Schmir, G. L. (1965). The effects of structural variation on the hydrolysis of $\Delta^{2}$-thiazolines. J Am Chem Soc 87, 2743-2751.

Schubert, S., Rakin, A., Karch, H., Carniel, E. \& Heesemann, J. (1998). Prevalence of the 'high-pathogenicity island' of Yersinia species among Escherichia coli strains that are pathogenic to humans. Infect Immun 66, 480-485.

Staggs, T. M. \& Perry, R. D. (1991). Identification and cloning of a fur regulatory gene in Yersinia pestis. J Bacteriol 173, 417-425.

Surgalla, M. J. \& Beesley, E. D. (1969). Congo red-agar plating medium for detecting pigmentation in Pasteurella pestis. Appl Microbiol 18, 834-837.

Venturi, V., Weisbeek, P. \& Koster, M. (1995). Gene regulation of siderophore-mediated iron acquisition in Pseudomonas: not only the Fur repressor. Mol Microbiol 17, 603-610.

Wake, A., Misawa, M. \& Matsui, A. (1975). Siderochrome production by Yersinia pestis and its relation to virulence. Infect Immun 12, 1211-1213.

Received 11 September 1998; revised 31 December 1998; accepted 21 January 1999. 